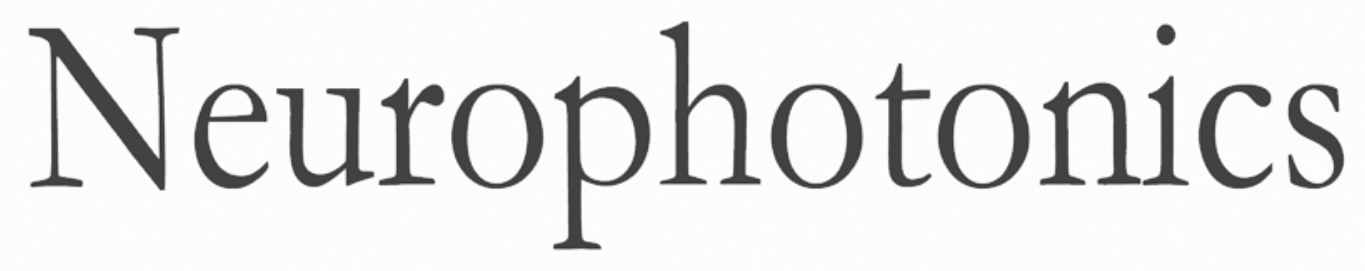

\title{
Bioluminescence imaging in live cells and animals
}

\author{
Jack K. Tung \\ Ken Berglund \\ Claire-Anne Gutekunst \\ Ute Hochgeschwender \\ Robert E. Gross
}




\title{
Bioluminescence imaging in live cells and animals
}

\author{
Jack K. Tung, ${ }^{a, b}$ Ken Berglund, ${ }^{b}$ Claire-Anne Gutekunst, ${ }^{b}$ Ute Hochgeschwender, ${ }^{c}$ and Robert E. Gross ${ }^{a, b, *}$ \\ ${ }^{a}$ Georgia Institute of Technology, Coulter Department of Biomedical Engineering, 313 Ferst Drive, Room 2127, Atlanta, Georgia 30332, \\ United States \\ bemory University, Department of Neurosurgery, 101 Woodruff Circle, WMRB Rm 6337, Atlanta, Georgia 30322, United States \\ ${ }^{\circ}$ Central Michigan University, College of Medicine and Neuroscience Program, Department of Neuroscience, $1280 \mathrm{~S}$. East Campus Street, \\ Mt. Pleasant, Michigan 48859, United States
}

\begin{abstract}
The use of bioluminescent reporters in neuroscience research continues to grow at a rapid pace as their applications and unique advantages over conventional fluorescent reporters become more appreciated. Here, we describe practical methods and principles for detecting and imaging bioluminescence from live cells and animals. We systematically tested various components of our conventional fluorescence microscope to optimize it for long-term bioluminescence imaging. High-resolution bioluminescence images from live neurons were obtained with our microscope setup, which could be continuously captured for several hours with no signs of phototoxicity. Bioluminescence from the mouse brain was also imaged noninvasively through the intact skull with a conventional luminescence imager. These methods demonstrate how bioluminescence can be routinely detected and measured from live cells and animals in a cost-effective way with common reagents and equipment. () The Authors. Published by SPIE under a Creative Commons Attribution 3.0 Unported License. Distribution or reproduction of this work in whole or in part requires full attribution of the original publication, including its DOI. [DOI: 10.1117/1.NPh.3.2.025001]
\end{abstract}

Keywords: bioluminescence; luciferase; imaging; live cell; in vivo.

Paper 15052TNR received Nov. 23, 2015; accepted for publication Feb. 23, 2016; published online Apr. 5, 2016.

\section{Introduction}

Conventional optical approaches for live cell imaging have generally relied on the use of various fluorescent proteins or synthetic molecules. These imaging techniques typically require an exogenous light source to excite the fluorescent molecules, where they enter a higher-energy state and subsequently emit light of a specific wavelength as they return to their ground state. ${ }^{1}$ Fluorescent molecules have proven to be exquisitely versatile reporters for live cell imaging because they span a broad spectrum of colors and can be detected with very high spatial and temporal resolution. Many important neuroscience questions regarding cellular anatomic structures, neuronal circuitry, molecular interactions, brain dynamics, and brain pathology have been addressed with the use of fluorescent molecules.

In contrast to fluorescence, bioluminescence is light generated from a chemical substrate and is routinely demonstrated in nature by various bioluminescent marine species, arthropods, fungi, and bacteria. ${ }^{2}$ These organisms generate light via an enzymatic reaction, in which a chemical substrate (e.g., luciferin) is oxidized by an enzyme (e.g., luciferase) ${ }^{2,3}$ Bioluminescence is therefore produced without any excitation light source and persists as long as the substrate is present. A variety of bioluminescent proteins spanning a broad spectrum of colors and emission properties have been identified and their genes cloned; the major ones used in neuroscience research are summarized in Table 1.

Bioluminescence imaging differs from fluorescent readouts in several aspects that, depending on the specific application, can be advantageous or disadvantageous. One major advantage of fluorescent molecules is that they can be far brighter than bioluminescent proteins; ${ }^{27}$ they can be made brighter by simply increasing the amount of excitation light, whereas bioluminescence intensity is strictly limited by the number of

*Address all correspondence to: Robert E. Gross, E-mail: rgross@emory.edu substrate molecules being catalyzed by the luciferase. Due to the relative dimness of bioluminescent proteins, longer exposure times are generally needed to collect a number of photons comparable to that of a fluorescence molecule. Bioluminescence imaging therefore generally has a limited temporal resolution compared to that of fluorescence imaging. ${ }^{27}$

On the other hand, several unique properties of bioluminescence make it an attractive imaging modality. First, bioluminescent signals generally have a higher signal-to-noise ratio (SNR). This is due to the fact that background luminescence is negligible compared to the signal produced from the luciferase reaction. ${ }^{28}$ Bioluminescent signals can therefore be much more sensitive than fluorescent signals, which generally have to compete with background auto-fluorescence. ${ }^{29}$ Second, bioluminescence does not require excitation light, eliminating the risk of photobleaching and phototoxicity that is associated with fluorescence imaging. ${ }^{30}$ Bioluminescent signals are therefore well suited for live cell imaging and can be recorded for much longer timescales compared to fluorescent signals without damaging reporter molecules or cells. Lastly, since bioluminescence requires no exogenous excitation light sources, it is a suitable optical readout for imaging light-sensitive cells such as retinal neurons.

Luciferase proteins have undergone significant evolution in their versatility as genetically encoded reporters for neuroscience research. Similar to their fluorescent counterparts, luciferase proteins can be targeted to specific regions in the cell with the use of trafficking or localization signal sequences to allow for imaging of subcellular structures over time. ${ }^{15}$ The concept of fluorescence resonance energy transfer has also been translated to bioluminescent proteins to measure molecular interactions. In this instantiation, both the intensity and spectral properties of bioluminescent proteins are altered when they are associated with fluorescent proteins in a process termed bioluminescence resonance energy transfer. ${ }^{31-33}$ Luciferase proteins have also 
Tung et al.: Bioluminescence imaging in live cells and animals

Table 1 Summary of major bioluminescent proteins used in neuroscience research.

\begin{tabular}{|c|c|c|c|c|c|c|}
\hline & Protein & Species & Emission peak & Mg, ATP? & Substrate & References \\
\hline \multirow[t]{4}{*}{ Luciferases } & FLuc & Firefly & 560 & $Y$ & D-Luciferin & $4-14$ \\
\hline & VLuc & Cypridina noctiluca & 460 & $Y$ & Vargulin luciferin & 5 \\
\hline & RLuc & Renilla reniformis & 480 & $\mathrm{~N}$ & Coelenterazine & $8,15-17$ \\
\hline & GLuc & Gaussia princeps & 480 & $\mathrm{~N}$ & Coelenterazine & $5,18,-21$ \\
\hline Photoproteins & Aequorin & Aequorea victoria & 470 & $\mathrm{~N}$ & Coelenterazine & $22-26$ \\
\hline
\end{tabular}

been engineered to respond to small molecules such as calcium and ATP, ${ }^{15}$ allowing them to be used for measuring changes in cellular dynamics such as neuronal activity. Protein engineering techniques have led to the development of brighter and longer wavelength luciferases that are well suited for in vivo imaging. ${ }^{34-38}$

Given the expanding toolbox of bioluminescent proteins and their wide variety of applications (see reviews by Badr and Tannous ${ }^{39}$ and Saito and Nagai ${ }^{40}$ ), it is timely to look at methods of detection of bioluminescence in laboratories not necessarily set up for bioluminescence imaging per se or unwilling to purchase commercially available bioluminescence imagers (e.g., Olympus LV200 microscope, IVIS Spectrum animal imager). In this paper, we discuss advantages and disadvantages of the various methods we have utilized for detection and quantification of bioluminescent signals from live cells and animals.

\section{Materials and Methods}

\subsection{Preparation of Coelenterazine Substrate}

The substrate for Renilla- and Gaussia-based luciferases, coelenterazine (CTZ), is typically dissolved in nonpolar solvents such as ethanol or methanol. These solvents are not ideal for live cell imaging due to their inherent toxicity. We therefore recommend solubilizing CTZ in aqueous solution with the help of inert chemical agents such as $\beta$-cyclodextrin as described by Teranishi and Shimomura ${ }^{41}$ or by utilizing commercially available solvents (e.g., Fuel-Inject from Nanolight Technology). Solutions of CTZ can be made at any desired concentration, although we routinely use a stock concentration of $600 \mu \mathrm{M}$ for in vitro use. After solubilization, CTZ can be aliquoted and stored at $-20^{\circ} \mathrm{C}$ for several months. Since the amount of CTZ needed for in vivo applications is relatively higher, we freshly prepare CTZ before each use following the manufacturer's recommendations. It is important to note that CTZ should be protected from light to prevent auto-oxidation.

\subsection{Cell Culture and Transfection}

HEK293 cells were passaged regularly in Dulbecco's Modified Eagle Medium (with $10 \% \mathrm{FBS}, 1 \%$ penicillin/streptomycin) and seeded to $90 \%$ confluency on glass cover slips the day before transfection. HEK cells were transfected with expression vectors encoding membrane-localized Renilla luciferase ${ }^{16}$ and membrane-localized Gaussia luciferase protein ${ }^{18}$ using Lipofectamine 2000 (Invitrogen). Transgene expression was confirmed by fluorescence microscopy the following day.

Dissociated cortical neuron cultures were derived from E18 rat embryos. Cortical tissue was digested with $2 \mathrm{mg} / \mathrm{mL}$ papain and dissociated by mechanical trituration before seeding onto 18-mm diameter German glass coverslips coated with $50 \mu \mathrm{g} / \mathrm{mL}$ poly-D-lysine (Sigma). Neuronal cultures were grown in serum-free Neurobasal media $(\mathrm{w} / 1 \times$ B27 supplement, $0.5 \mathrm{mM}$ glutamine) and media was changed (half-volume) every 3 to 4 days. Cortical neuron cultures were transduced with viral vectors encoding luciferase 2 to 3 days after plating.

\subsection{Live Cell Bioluminescence Imaging}

Bioluminescence images were taken on an Olympus inverted fluorescence microscope equipped with a variety of objectives, c-mount adaptors, and cameras (Table 2).

Cells cultured on glass coverslips were transferred to a perfusion chamber (Warner Instruments) containing phenol-free media at the time of imaging to minimize light absorption and maximize transmission of bioluminescence through the media (this is especially important for upright microscopes). The cells were then checked under fluorescence to confirm transgene expression and determine the right depth of focus for bioluminescence imaging. The microscope was then switched to an empty filter position to collect whole-spectrum bioluminescence.

Images were collected using the open-source Micromanager image acquisition software. Camera settings were standardized and optimized by cooling the chip to the lowest temperature (to minimize dark current) and maximizing the gain. Binning was used only when it was necessary to produce visible images. All of the background light sources in the room (windows, doors,

Table 2 Objectives, adaptors, and cameras tested.

\begin{tabular}{|c|c|c|}
\hline Objectives & Adaptors & Cameras \\
\hline PlanApo $60 \times$ oil, $1.4 \mathrm{NA}$ & $0.5 \times$ & $\begin{array}{l}\text { Coolsnap-ES CCD } \\
\text { (Photometrics) }\end{array}$ \\
\hline UPlanFI 40× oil, 1.3NA & $0.3 x$ & $\begin{array}{l}\text { Coolsnap-FX CCD } \\
\text { (Photometrics) }\end{array}$ \\
\hline LUMPlanFLN W $60 \times, 1.0 \mathrm{NA}$ & & $\begin{array}{c}\text { ImagEM C9100-13 } \\
\text { EMCCD (Hamamatsu) }\end{array}$ \\
\hline UPlanSApo 20×, 0.75NA & & $\begin{array}{l}\text { iXon Ultra } 897 \text { EMCCD } \\
\text { (Andor) }\end{array}$ \\
\hline \multirow[t]{2}{*}{ LucPlanFL 60×, 0.7NA } & & $\begin{array}{l}\text { QuantEM EMCCD } \\
\text { (Photometrics) }\end{array}$ \\
\hline & & $\begin{array}{l}\text { OptiMOS sCMOS } \\
\text { (Photometrics) }\end{array}$ \\
\hline
\end{tabular}


and electronics) were covered with blackout material and images were collected at various exposure times (1 to $40 \mathrm{~s}$ ). Long-term bioluminescence images were acquired using the multiacquisition feature of Micromanager and a perfusion system to deliver CTZ during the imaging period (up to $3 \mathrm{~h}$ ). Imaging was done under controlled conditions that included using the same batch of transfected cells, CTZ concentration, media, exposure times, and imaging conditions to reduce variability between experiments. We did not attempt to normalize bioluminescence signals between different sets of experiments, although we employed the same camera settings (i.e., exposure time and binning) for a given combination of the microscope, camera, objective lens, and luciferase used. Thus, the bioluminescence signal was comparable within a given set of the experiments.

For the comparison of camera models in Fig. 3, bioluminescence imaging was repeated using an upright microscope (Eclipse E600-FN, Nikon) and a water-immersion objective lens (40×, NA 0.8). CTZ was applied to the same batch of HEK cells transiently transfected with a membrane-bound Gaussia luciferase cultured on glass coverslips. Images were taken every $5 \mathrm{~s}$ with exposure time of $4.5 \mathrm{~s}$ and CTZ $(100 \mu \mathrm{M})$ was bath-applied $50 \mathrm{~s}$ after the start of an imaging session. The camera models compared were Photometrics CoolSNAP ES [noncooled scientific charge-coupled device (CCD); digitization: 12 bit; gain $0.3 \times$ ], Photometrics CoolSNAP fx (cooled scientific CCD; digitization: 12 bit; gain $2 \times$ ), Hamamatsu ImagEM C9100-13 (EMCCD; digitization: 16 bit; EM gain: 1200×), and Andor iXon Ultra 897 (EMCCD; digitization: 16 bit; EM gain $1000 \times)$. Each camera was binned to roughly $256 \times 256$ pixels, resulting in a similar pixel size of $25.8 \mu \mathrm{m}$ (CoolSNAP ES), $26.8 \mu \mathrm{m}$ (CoolSNAP fx), $32 \mu \mathrm{m}$ (ImagEM), and $32 \mu \mathrm{m}$ (iXon Ultra 897).

Image quality was estimated by calculating the SNR of captured images at the peak luminescence time point. SNR was calculated by dividing the mean pixel intensity of the bioluminescence image to the standard deviation of the pixel intensity from a background image (or region with no cells) in ImageJ.

\subsection{In Vivo Bioluminescence Imaging}

For imaging the mouse brain, an adeno-associated virus (AAV) encoding Renilla luciferase (RLuc) was injected into the cortex (-1.58 AP, $-0.75 \mathrm{ML},-0.8 \mathrm{SI}$, and $-1.58 \mathrm{AP},-1.75 \mathrm{ML}$, $-0.5 \mathrm{SI}$ ) of 5- to 6-week old CD1 white mice (Harlan). After 2 weeks, the fur was shaved off the head and the animals were anesthetized with ketamine/xylazine before bioluminescence imaging. $500 \mu \mathrm{g}$ of CTZ was administered either intraperitoneally or intravenously (via tail vein injection). Bioluminescence images were captured with the animals still under ketamine/xylazine anesthesia using a conventional luminescence imager (Fuji LAS-3000). Sequential images of $20 \mathrm{~s}$ exposure time were captured and the total signal intensity of each image was quantified in ImageJ to achieve a bioluminescence signal time course. Images at the time of maximum signal intensity are displayed showing the relative bioluminescence signal pseudocolored by a RGB lookup table in ImageJ. All procedures were performed in accordance with the US National Institutes of Health guidelines for animal research and were approved by the Institutional Animal Care and Use Committee at Emory University.

\section{Results and Discussion}

\subsection{Live Cell Bioluminescence Imaging}

Image brightness is directly related to the light-gathering power of the objective (numerical aperture, NA) and inversely related to the image magnification (M): brightness $\propto(\mathrm{NA} / \mathrm{M}) .{ }^{2}$ We have therefore maximized the image brightness of our samples by optimizing three components of our microscope: the objective, camera, and intermediate optics.

In selecting an appropriate objective lens for bioluminescence imaging, we aimed at finding one with the highest NA and lowest workable magnification. In comparing various objective lenses, we found that objectives with higher NA produced brighter, higher resolution images [Figs. 1(a2) versus 1(a1)]. Lower magnification objectives were also able to produce higher resolution images [Figs. 1(b2) versus 1(b1)].

We utilized an intermediate demagnifying lens on a camera mount in order to allow more light to be focused onto a smaller area of the camera chip. Each pixel in the illuminated area of the chip therefore receives more light, producing a brighter image. We found that greater demagnification produced brighter images with sufficient spatial resolution to visualize detailed cellular morphology [Figs. 2(a) versus 2(b)]. Note that silhouetting at the periphery of the image may occur with greater demagnification, so the field of view requirements must be carefully considered.

There are numerous camera options currently available for low-light optical imaging. CCD cameras are the most popular choice and rely on the photoelectric effect to convert a light signal into an electrical signal. The readout noise of CCD devices was significantly reduced with the advent of electron multiplying charge-coupled devices (EMCCD), making these cameras
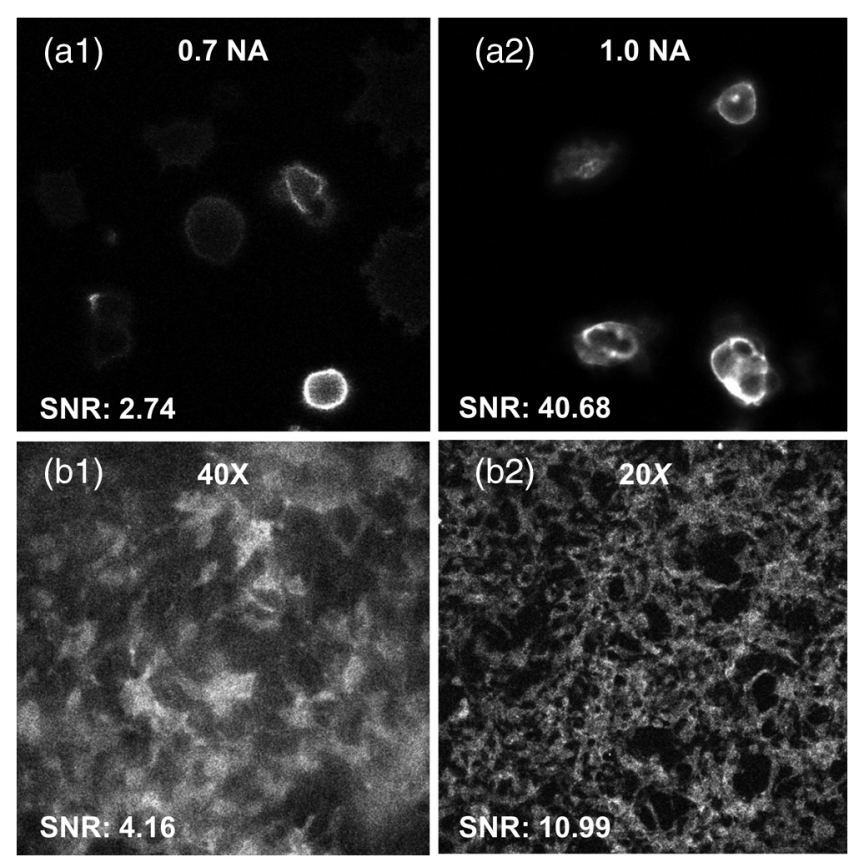

Fig. 1 Objective lens comparison. Bioluminescence images from cultured HEK cells expressing Gaussia luciferase (a1 and a2) and Renilla luciferase (b1 and b2) with the following objectives: (a1) LUCPlanFL 60× 0.7 NA, (a2) LUMPlanFLN/W 60× 1.0 NA for comparison of NA, (b1) UPlanFI 40x oil 1.3NA, and (b2) UPlanSApo 20x 0.75 NA for comparison of different magnification together with NA. 

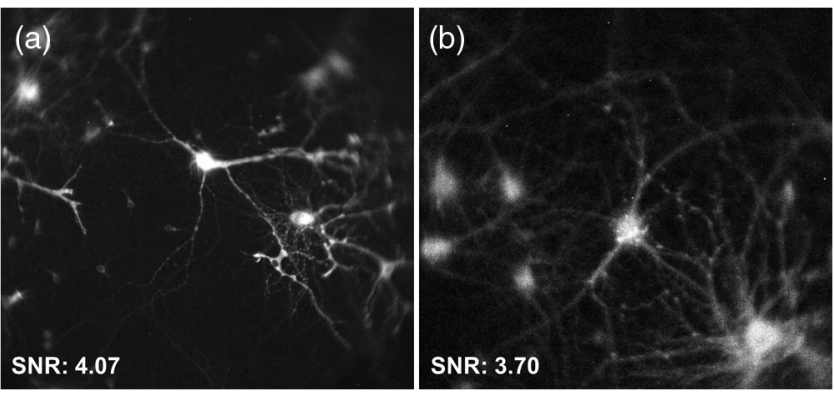

Fig. 2 Intermediate lens comparison. Bioluminescence images of dissociated cortical neuron cultures expressing Renilla luciferase using a $0.3 \times$ intermediate lens (a) and a $0.5 \times$ intermediate lens (b). Both images were taken with an UAPO $40 \times 1.35$ NA oil objective and a sCMOS (OptiMOS, Photometrics) camera.

especially well suited for low-light imaging applications. Scientific CMOS (sCMOS) devices are a relatively new type of sensor that also offers extremely low readout noise and wide dynamic range, making them a cost-effective alternative for imaging in low-light conditions. We have therefore compared several CCD, EMCCD, and sCMOS cameras for bioluminescence imaging (Fig. 3). All of the cameras tested were able to produce bioluminescence images with relatively low background and short exposure times ( 1 to $10 \mathrm{~s}$ ). Even though EMCCD cameras generally outperform sCMOS devices at very low light levels, our images were qualitatively similar (most likely due to the fact that RLuc and GLuc are relatively bright luciferases). One should therefore select a camera based on the level of sensitivity required, as the price differences between EMCCD, sCMOS, and CCD cameras can be quite significant.
Due to the relatively long-exposure times required for bioluminescence imaging, we found that it was important to reduce the amount of ambient light in the room as much as possible to reduce background noise. We routinely turned off or covered light sources (such as an arc lamp for fluorescence observation) near the microscope before bioluminescence imaging. We found that blackout curtains were especially effective at isolating the microscope and camera from any potential light contamination. Cooling the camera to the lowest temperature setting also helped reduce background by limiting dark current noise.

The danger of phototoxicity and photobleaching limits the effectiveness of long-term live-cell imaging with fluorescent molecules. In contrast, we have demonstrated that bioluminescent reporters can be used to image live cells for extended periods of time without any apparent adverse effects (Fig. 4). The substrate was supplied by a perfusion system, resulting in a long-lasting bioluminescent signal which could be detected over several hours. We did not observe any apparent adverse effects during this period, making this approach suitable for imaging cellular processes that are directed over long timescales, such as cellular trafficking, synaptogenesis, and migration.

\subsection{In Vivo Bioluminescence Imaging}

In vivo fluorescence imaging is often compromised by high nonspecific background from tissue and cells (auto-fluorescence). In contrast, background bioluminescence from tissues not expressing luciferase is negligible. This property of bioluminescence makes it an ideal signal for imaging whole animals where the number of luciferase-expressing target cells is generally few compared to the surrounding non-expressing tissue. We assessed the usability of Renilla luciferase for in vivo imaging
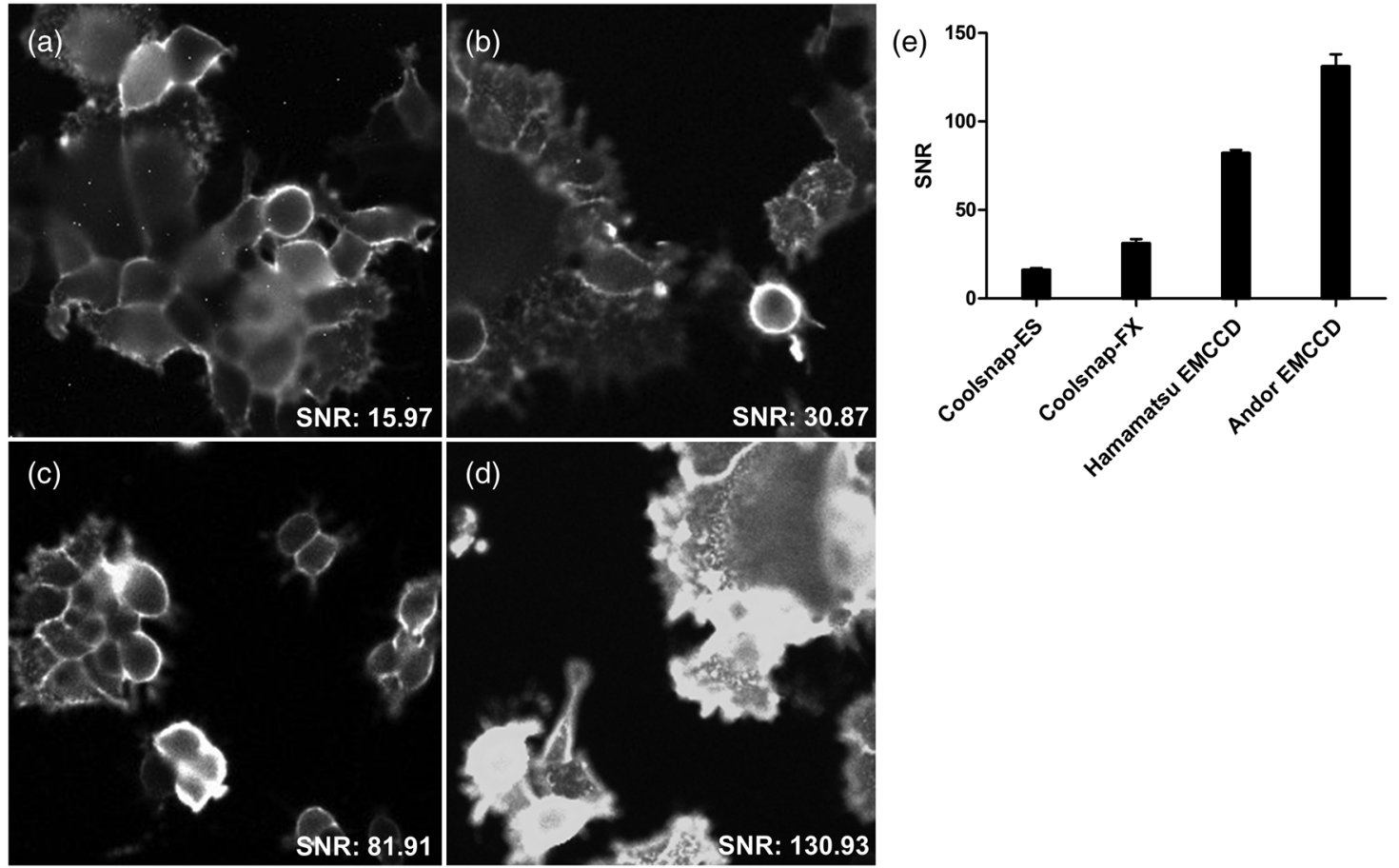

Fig. 3 Camera comparison. Bioluminescence images of HEK293 cells transfected with Gaussia luciferase taken with various cameras: (a) Noncooled scientific CCD (Coolsnap-ES, Photometrics); (b) cooled scientific CCD (Coolsnap-FX, Photometrics); (c): EMCCD (ImagEM C9100-13, Hamamatsu); (d): EMCCD (iXon Ultra 897, Andor). Contrast was adjusted for each camera using the highest and lowest intensity obtained in each camera. (e) Average SNR calculated at the time of peak luminescence for each camera. 


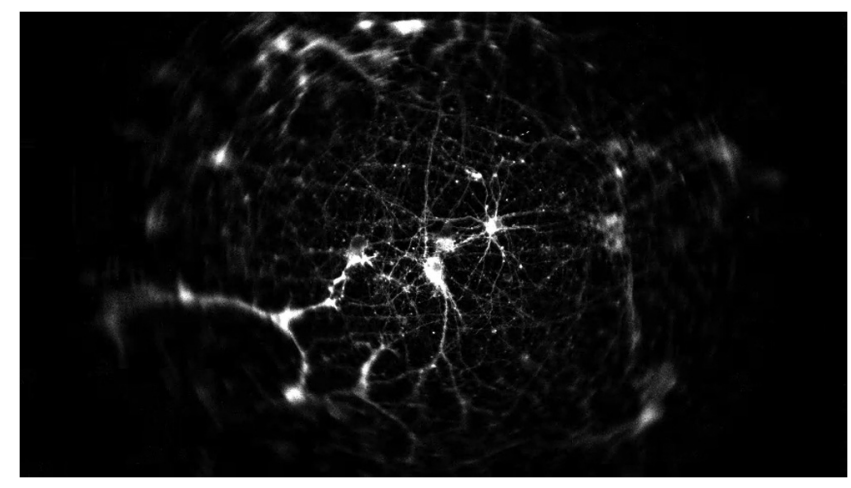

Fig. 4 Long-term bioluminescence imaging in vitro. Time-lapse bioluminescence images illustrate the ability to conduct live cell imaging over extended periods of time without the threat of phototoxicity or photobleaching. Cortical neurons expressing Renilla luciferase were imaged over a period of $3 \mathrm{~h}$. Note that the bioluminescent signal decreases over time (as substrate availability decreases) but is able to be refreshed again by the addition of more substrate. (Video 1, WMV, 1014 KB) [URL: http://dx.doi.org/10.1117/1.NPh.3.2.025001.1].

and found that the bioluminescence signal was strong enough to allow detection of signals through the intact skull [Figs. 5(a) and 5(b)]. Special consideration of the route of substrate administration is needed when selecting a luciferase to use for in vivo bioluminescence imaging. Firefly luciferase (Fluc) has been frequently used for bioluminescence imaging in animals due to the relatively ease of the intraperitoneal route of substrate (D-luciferin) administration. Although we have demonstrated that coelenterazine can also be delivered intraperitoneally, Renilla luciferase is maximally effective when the substrate is administered via intravenous routes [Fig. 5(c)]. This may be

(a)

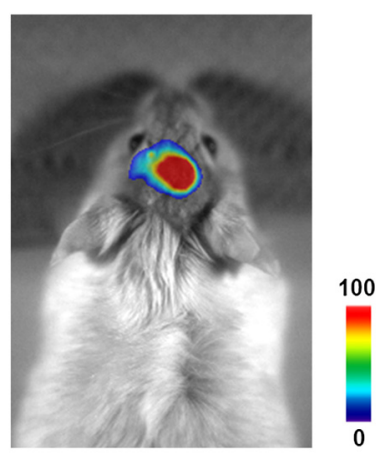

(c)

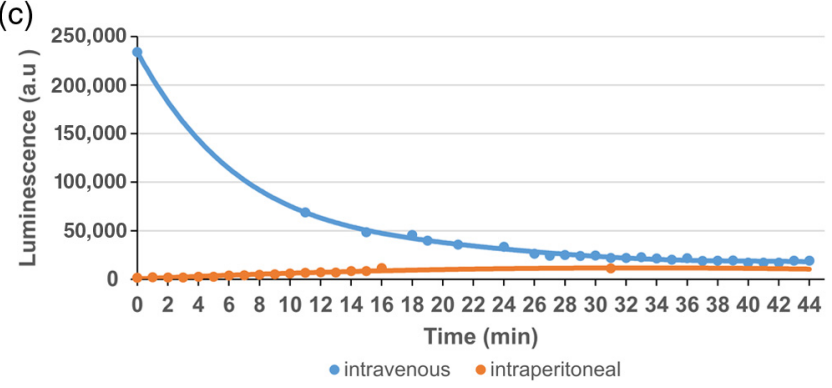

Fig. 5 In vivo bioluminescence imaging. Bioluminescence imaging of an adult mouse injected with AAV encoding Renilla luciferase in the cortex 2 weeks prior. $500 \mu \mathrm{g} \mathrm{CTZ}$ was delivered to the same mouse either intravenously (a) or intraperitoneally (b). Color bar depicts relative luminescent signal. (c) Bioluminescence images were taken over time and quantified in ImageJ to create a plot of bioluminescence time course. due to the fact that the bioavailability of substrate in the brain is reduced when it is administered intraperitoneally, where it needs to drain through the lymphatic system before entering the bloodstream. Since beetle (e.g., firefly) and marine (Renilla, Gaussia) luciferases utilize different luciferin substrates, it is therefore feasible to multiplex them together due to absence of crosstalk between the two systems.

The low background with bioluminescence makes in vivo bioluminescence imaging particularly sensitive for detecting signals from small areas over extended periods of time. In fact, bioluminescence imaging has been successfully used for applications from tracking transplanted cells ${ }^{42,43}$ to monitoring cellular processes such as neurodegeneration, inflammation, and neurogenesis. ${ }^{44}$ Although in vivo bioluminescence signals provide limited spatial information directly, the cell-type specificity of luciferase expression provides indirect spatial information because any detected bioluminescence should ostensibly be coming only from cells expressing luciferase.

\section{Conclusion}

We have demonstrated how bioluminescent proteins can be effectively used as an optical reporter for both in vitro and in vivo settings. Given the proper detection equipment and scientific question, bioluminescence offers several unique advantages over conventional fluorescence readouts. The major advantage of using bioluminescent signals is that they are detected over little to no background noise, enabling long term imaging applications with no risk of phototoxicity or artifact. With the development of brighter and more responsive luciferase proteins, the use of bioluminescent reporters in neuroscience research will continue to grow more robust and versatile.

\section{Acknowledgments}

Funding sources: NS079268 and NS079757 to R.E.G.; NS086433 to J.K.T.; NSF 1512826 to K.B.; Duke Institute for Brain Science (DIBS Research Incubator Award) and MH101525 to U.H.

\section{References}

1. J. W. Lichtman and J.-A. Conchello, "Fluorescence microscopy," Nat. Methods 2, 910-919 (2005).

2. J. W. Hastings, "Bioluminescence," Annu. Rev. Cell Dev. Biol. 14, 197-230 (1998).

3. M. Keyaerts, V. Caveliers, and T. Lahoutte, "Bioluminescence imaging: looking beyond the light," Trends Mol. Med. 18, 164-172 (2012).

4. M. Aswendt et al., "Boosting bioluminescence neuroimaging: an optimized protocol for brain studies," PLoS One 8, e55662 (2013).

5. C. A. Maguire et al., "Triple bioluminescence imaging for in vivo monitoring of cellular processes," Mol. Ther. Nucleic Acids 2, e99 (2013).

6. M. S. Evans et al., "A synthetic luciferin improves bioluminescence imaging in live mice," Nat. Methods 11(4), 393-395 (2014).

7. H. Im et al., "In vivo visualization and monitoring of viable neural stem cells using noninvasive bioluminescence imaging in the 6-hydroxydopamine-induced mouse model of Parkinson disease," Mol. Imaging 12.4, 224-234 (2013).

8. K. Shah, "Imaging neural stem cell fate in mouse model of glioma," Curr. Protoc. Stem Cell Biol. 5A.1 (2009).

9. J. Bakhsheshian et al., "Bioluminescent imaging of drug efflux at the blood-brain barrier mediated by the transporter ABCG2," Proc. Natl. Acad. Sci. U. S. A. 110, 20801-20806 (2013).

10. L. Zhu et al., "Non-invasive imaging of GFAP expression after neuronal damage in mice," Neurosci. Lett. 367, 210-212 (2004).

11. J. C. Watts et al., "Bioluminescence imaging of Abeta deposition in bigenic mouse models of Alzheimer's disease," Proc. Natl. Acad. Sci. U. S. A 108, 2528-2533 (2011). 
12. A. F. Keller, M. Gravel, and J. Kriz, "Live imaging of amyotrophic lateral sclerosis pathogenesis: disease onset is characterized by marked induction of GFAP in Schwann cells," Glia 57, 1130-1142 (2009).

13. D. W. Hwang et al., "Noninvasive in vivo monitoring of neuronal differentiation using reporter driven by a neuronal promoter," Eur. J. Nucl. Med. Mol. Imaging 35, 135-145 (2008).

14. H. J. Oh et al., "In vivo bioluminescence reporter gene imaging for the activation of neuronal differentiation induced by the neuronal activator neurogenin 1 (Ngn1) in neuronal precursor cells," Eur. J. Nucl. Med. Mol. Imaging 40, 1607-1617 (2013).

15. K. Saito et al., "Luminescent proteins for high-speed single-cell and whole-body imaging," Nat. Commun. 3, 1262 (2012).

16. J. K. Tung, C.-A. Gutekunst, and R. E. Gross, "Inhibitory luminopsins: genetically-encoded bioluminescent opsins for versatile, scalable, and hardware-independent optogenetic inhibition," Sci. Rep. 5, 14366 (2015).

17. A. Takai et al., "Expanded palette of nano-lanterns for real-time multicolor luminescence imaging," 112, 4352-4356 (2015).

18. K. Berglund et al., "Light-emitting channelrhodopsins for combined optogenetic and chemical-genetic control of neurons," PLoS One $\mathbf{8}$, e59759 (2013).

19. B. A. Tannous et al., "Codon-optimized Gaussia luciferase cDNA for mammalian gene expression in culture and in vivo," Mol. Ther. 11, 435-443 (2005).

20. J. M. Niers et al., "Single reporter for targeted multimodal in vivo imaging," J. Am. Chem. Soc. 134, 5149-5156 (2012).

21. K. Berglund et al., "Luminopsins integrate opto- and chemogenetics by using physical and biological light sources for opsin activation," Proc. Natl. Acad. Sci. U. S. A. 113, E358-E367 (2016).

22. A. Bakayan et al., "Red fluorescent protein-aequorin fusions as improved bioluminescent $\mathrm{Ca} 2+$ reporters in single cells and mice," PLoS One 6, e19520 (2011).

23. T. Curie et al., "Red-shifted aequorin-based bioluminescent reporters for in vivo imaging of Ca2 signalling," Mol. Imaging 6.1, 30 (2007).

24. V. Baubet et al., "Chimeric green fluorescent protein-aequorin as bioluminescent Ca2+ reporters at the single-cell level," Proc. Natl. Acad. Sci. U. S. A. 97.3, 7260-7265 (2000).

25. K. L. Rogers et al., "Visualization of local $\mathrm{Ca} 2+$ dynamics with genetically encoded bioluminescent reporters," Eur. J. Neurosci. 21, 597-610 (2005).

26. E. Drobac et al., "Calcium imaging in single neurons from brain slices using bioluminescent reporters," J. Neurosci. Res. 88, 695-711 (2010).

27. G. Choy et al., "Comparison of noninvasive fluorescent and bioluminescent small animal optical imaging," Biotechniques 35(5), 1022-1030 (2003).

28. T. Troy et al., "Quantitative comparison of the sensitivity of detection of fluorescent and bioluminescent reporters in animal models," Mol. Imaging 3, 9-23 (2004).

29. C. H. Contag, Molecular Imaging: Principles and Practice, R. R. B. Weissleder, Ed., pp. 118-138, People's Medical Publishing House, Shelton, Connecticut (2010).

30. N. Billinton and A. W. Knight, "Seeing the wood through the trees: a review of techniques for distinguishing green fluorescent protein from endogenous autofluorescence," Anal. Biochem. 291, 175-197 (2001).

31. A. Dragulescu-andrasi et al., "Bioluminescence resonance energy transfer (BRET) imaging of protein- protein interactions within deep tissues of living subjects," Proc. Natl. Acad. Sci. U. S. A. 108(29), 12060-12065 (2011).

32. A. De et al., "Evolution of BRET biosensors from live cell to tissuescale in vivo imaging," Front. Endocrinol. 4, 131 (2013).

33. Z. Xia and J. Rao, "Biosensing and imaging based on bioluminescence resonance energy transfer," Curr. Opin. Biotechnol. 20, 37-44 (2009).

34. S. B. Kim et al., "Superluminescent variants of marine luciferases for bioassays," Anal. Chem. 83(22), 8732-8740 (2011).

35. B. R. Branchini et al., "Red-emitting luciferases for bioluminescence reporter and imaging applications," Anal. Biochem. 396, 290-297 (2010).

36. A. M. Loening, A. M. Wu, and S. S. Gambhir, "Red-shifted Renilla reniformis luciferase variants for imaging in living subjects," Nat. Methods 4, 641-643 (2007).
37. A. M. Loening, A. M. Wu, and S. S. Gambhir, "Red-shifted Renilla reniformis luciferase variants for imaging in living subjects," Nat. Methods 4.8 (2007).

38. B. R. Branchini et al., "Red- and green-emitting firefly luciferase mutants for bioluminescent reporter applications," Anal. Biochem. 345, 140-148 (2005).

39. C. E. Badr and B. A. Tannous, "Bioluminescence imaging: progress and applications," Trends Biotechnol. 29.12, 624-633 (2011).

40. K. Saito and T. Nagai, "Recent progress in luminescent proteins development," Curr. Opin. Chem. Biol. 27, 46-51 (2015).

41. K. Teranishi and O. Shimomura, "Solubilizing coelenterazine in water with hydroxypropyl cyclodextrin," Biosci. Biotech. Biochem. 61, 1219-1220 (1997).

42. L. Mezzanotte et al., "Evaluating reporter genes of different luciferases for optimized in vivo bioluminescence imaging of transplanted neural stem cells in the brain," Contrast Media Mol. Imaging 8, 505-513 (2013).

43. A. Tennstaedt et al., "Noninvasive multimodal imaging of stem cell transplants in the brain using bioluminescence imaging and magnetic resonance imaging," Methods Mol. Biol. 1052, 153-166 (2013).

44. K. Hochgräfe and E.-M. Mandelkow, "Making the brain glow: in vivo bioluminescence imaging to study neurodegeneration," Mol. Neurobiol. 47, 868-882 (2013).

Jack K. Tung is an MD/PhD student currently pursuing his $\mathrm{PhD}$ in biomedical engineering at Georgia Institute of Technology and Emory University. His current research interests include developing and applying novel technologies for the treatment of neurological disorders. His dissertation comprised developing novel optogenetic probes driven by bioluminescence (inhibitory luminopsins) and applying them in various animal models of epilepsy to control seizure activity.

Ken Berglund is a research associate at Emory University School of Medicine. His research focus is development of novel optogenetic tools and application of his tools in studies of neurophysiology and neuropathology in the rodent brain.

Claire-Anne Gutekunst received her $\mathrm{PhD}$ in psychobiology and is currently assistant professor in Neurosurgery, Emory School of Medicine. She is a behavioral scientist and anatomist who specializes in developing and validating gene therapies for various CNS disorders. She has developed a gene therapy for neurodegenerative diseases using C3 transferase, a bacterial exoenzyme with RhoA inhibition activity. She uses $\mathrm{C} 3$ expressing vectors to promote axon regrowth in the otherwise nonpermissive adult CNS with applications to various disorders including optic nerve injury, glaucoma, spinal cord injury, stroke, and other neurodegenerative disorders.

Ute Hochgeschwender, MD, is currently an associate professor of neuroscience at Central Michigan University's College of Medicine. Her long-term interest has been the molecular basis of brain function resulting in behavior. Over the past several years she developed a method to integrate the optogenetic and chemogenetic analyses of neural circuits by combining optogenetics with bioluminescence. Her current research interests are in further advancing the concept of bioluminescence-driven optogenetics and in utilizing these tools for research into the mechanisms and potential, noninvasive treatment of neurodegenerative and psychiatric diseases.

Robert E. Gross, MD, PhD, is the MBNA/Bowman Endowed Chair in Neurosurgery at Emory University School of Medicine, with appointments in Neurology and the Coulter Department of Biomedical Engineering at Emory/Georgia Institute of Technology. He is a neurosurgeon specialized in functional neurosurgery, and director of the Emory Neuromodulation Technology Innovation Center (ENTICe). He runs the Translational Neuroengineering Research Lab, developing and refining new treatments for neurological and psychiatric disorders, using molecular and electrical techniques for neuromodulation, including novel optogenetic techniques. 